\title{
Isosceles triple convexity
}

\section{LIPING YUAN ${ }^{1}$, TUDOR ZAMFIRESCU ${ }^{2,3}$ and YUE ZHANG ${ }^{1}$}

\section{ABSTRACT.}

A set $S$ in $\mathbb{R}^{d}$ is called $i t$-convex if, for any two distinct points in $S$, there exists a third point in $S$, such that one of the three points is equidistant from the others.

In this paper we first investigate nondiscrete $i t$-convex sets, then discuss about the $i t$-convexity of the eleven Archimedean tilings, and treat subsequently finite subsets of the square lattice. Finally, we obtain a lower bound on the number of isosceles triples contained in an $n$-point $i t$-convex set.

Acknowledgements. Thanks are due to one referee for his pertinent remarks.

The first author gratefully acknowledges financial support by NSF of China (11471095), NSF of Hebei Province (A2012205080, A2013205189), Program for Excellent Talents in University, Hebei Province (GCC2014043).

The second author thankfully acknowledges the financial support by the High-end Foreign Experts Recruitment Program of People's Republic of China. His work was also partly supported by a grant of the Roumanian National Authority for Scientific Research, CNCS -UEFISCDI, project number PN-II-ID-PCE-2011-3-0533.

\section{REFERENCES}

[1] Blind, R., Valette, G. and Zamfirescu, T., Rectangular convexity, Geom. Dedicata, 9 (1980), 317-327

[2] Böröczky, Jr. K., Rectangular convexity of convex domains of constant width, Geom. Dedicata, 34 (1990), 13-18

[3] Grünbaum, B. and Shephard, G. C., Tilings and Patterns. New York: W. H. Freeman and Company, 1987

[4] Magazanik, E. and Perles, M. A., Staircase connected sets, Discrete Comp. Geom., 37 (2007), 587-599

[5] Olds, C. D., Lax, A. and Davidoff, G. P., The Geometry of Numbers, Washington, DC: The Mathematical Association of Anerica, 2000

[6] Yuan, L. and Zamfirescu, T., Right triple convex completion, J. Convex Analysis, 22 (2015), No. 1, 291-301

[7] Yuan, L. and Zamfirescu, T., Right triple convexity, J. Convex Analysis, 23 (2016), No. 4, 1219-1246

[8] Zamfirescu, T., Right Convexity, J. Convex Analysis, 21 (2014), 253-260

\author{
${ }^{1}$ Hebei Normal UNIVERSITY \\ COLlEGE OF MATHEMATICS AND INFORMATION SCIENCE \\ NANerhuAn EASt RoAd 20, 050024, ShijIAZHuANG, P.R. CHINA \\ E-mail address: lpyuan@hebtu.edu. cn \\ E-mail address: zhangyue0309@126.com
}

${ }^{2}$ TU DORTMUND

FACHBEREICH MATHEMATIK

VOGELPOTHSWEG 87, 44221 DORTMUND, GERMANY

E-mail address: tudor.zamfirescu@mathematik.uni-dortmund.de

Received: 11.04.2015; In revised form: 27.02.2016; Accepted: 21.04.2016

2010 Mathematics Subject Classification. 53C45, 53C22.

Key words and phrases. it-convex, Archimedean tiling, monotone set, poor n-point it-convex set.

Corresponding author: Liping Yuan; lpyuan@hebtu.edu.cn 
${ }^{3}$ ROUMANIAN ACADEMY

Institute of MATHEMATics "Simion Stoilow"

P.O. BOX 1-764, RO-014700, BUCHAREST, ROUMANIA

E-mail address: tudor.zamfirescu@mathematik.uni-dortmund.de 